\title{
Die Handtherapie in der Behandlung des CRPS
}

\author{
Hand Therapy in the Treatment of Patients with CRPS
}

Autoren

Institute

\section{Körbler', M. Pfau², F. Becker², U. Koester ${ }^{3}$, F. Werdin}

${ }^{1}$ Abteilung für Physiotherapie und Ergotherapie, Marienhospital Stuttgart, Stuttgart

${ }^{2}$ Abteilung für Plastische Chirurgie und Handchirurgie, Diakonieklinkum Stuttgart, Stuttgart

${ }^{3}$ Klinik für Anästhesiologie und Operative Intensivmedizin, Spezielle Schmerztherapie, Marienhospital Stuttgart, Stuttgart

\author{
Schlüsselwörter \\ - CRPS \\ - komplex regionales \\ Schmerzsyndrom \\ - Handtherapie \\ - Rehabilitation \\ Key words \\ - CRPS \\ complex regional pain \\ syndrome \\ - hand therapy \\ - rehabilitation
}

eingereicht $\quad 2.10 .2014$

akzeptiert $\quad 7.2 .2015$

Bibliografie

DOI http://dx.doi.org/

10.1055/s-0035-1547302

Handchir Mikrochir Plast Chir

2015; 47: 182-189

(c) Georg Thieme Verlag KG

Stuttgart · New York

ISSN 0722-1819

Korrespondenzadresse

\section{Dr. Frank Werdin}

Abteilung für Plastische Chirurgie und Handchirurgie Diakonieklinikum Stuttgart

Rosenbergstraße 38

70176 Stuttgart

fwerdin@gmx.de

\section{Zusammenfassung}

$\nabla$

In der modernen Therapie des komplexen regionalen Schmerzsyndroms (CRPS) hat sich ein multimodales Behandlungskonzept (MMST: multimodale Schmerztherapie) durchgesetzt. Neben der medikamentösen Therapie und der Psychotherapie zählen die Physio- und Ergotherapie (zusammen Handtherapie) zu den Basistherapieformen. Trotz dieses hohen Stellenwertes innerhalb der Behandlung des CRPS sind die einzelnen Anwendungsformen der Handtherapie nur wenig wissenschaftlich untersucht und Ihr Einsatz kaum standardisiert. Ziel der vorliegenden Arbeit war es daher, die in der Behandlung des CRPS zur Anwendung kommenden handtherapeutischen Techniken hinsichtlich Funktion/Durchführung, Anwendung und Wirksamkeit darzustellen und einen entsprechenden, einheitlichen Behandlungsalgorithmus daraus abzuleiten. Hierzu wurden sämtliche in der Therapie eingesetzten Anwendungen dargestellt und bzgl Ihrer Wirksamkeit mittels Literaturrecherche überprüft. Ein erwiesener Nutzen konnte bei der Bewegungstherapie, der Manuellen Therapie, dem Graded Motor Imaging/Spiegeltherapie, den Kohlensäurebädern und der Ergotherapie festgestellt werden. Obwohl harte, evidenz-basierte Daten für viele Anwendungen fehlen, konnte ein Behandlungsalgorithmus formuliert werden. Es bedarf jedoch dringend weiterer Studien hinsichtlich der Wirksamkeit als auch der Anwendungshäufigkeit und Intensität handtherapeutischer Anwendungen innerhalb der Behandlung des CRPS.

\section{Einleitung $\nabla$}

Das komplexe regionale Schmerzsyndrom (CRPS) wird aktuell definiert als: „...eine anhaltende Empfindungsstörung (Schmerz, Hyperästhesie, Hyperalgesie, Allodynie) auf ein initial schädi-

\section{Abstract \\ $\nabla$}

In the modern treatment of CRPS a multidisciplinary concept is firmly established (MMPT, multimodal pain therapy). Besides medical therapy and psychotherapy, physio- and occupational therapy count as basic treatment options. Although physio- and occupational therapy (in the following called hand therapy) are the most important basic treatments, the therapy is hardly standardised and there are few scientific investigations concerning their application. Therefore the purpose of this paper is to present the applied hand therapeutic techniques with regard to function/performance, application and effectiveness, and to derive a suitable treatment algorithm. The techniques used in hand therapy are presented and reviewed in regard to their effectiveness by means of a literature search. It turns out that exercise therapy, manual therapy, graded motor imaging, $\mathrm{CO}_{2}$ baths and occupational therapy have a proven benefit for the patients. Although for many of the treatments reliable evidence-based data are lacking a treatment algorithm was established but there is a strong need for further investigations concerning the therapeutic effectiveness in the treatment of CRPS.

gendes Ereignis, nicht auf das Versorgungsgebiet eines peripheren Nerven und auch nicht auf den ursprünglich geschädigten Ort beschränkt, in der Regel bis distal zu den Akren reichend, und unverhältnismäßig zum Trauma“ [1]. Die Erkrankung kann in 3 Stadien eingeteilt werden 
Tab. 1 Stadieneinteilung des CRPS nach Sudeck:

Stadium I: Akutes, bzw. Entzündungsstadium (bis zu 3 Monaten)
Im Vordergrund stehen Schmerzen (auch Ruheschmerz), Ödematisierung
und Funktionseinschränkung. Die Haut ist livide verfärbt und teigig
verändert sowie meist überwärmt.
Stadium II: Dystrophie ( 3 Monate bis ein Jahr)
Gewebsatrophie, häufig lässt der Schmerz nach, gelegentlich kommt es zu
einer Abkühlung der Haut.
Stadium III: Atrophie (ab einem Jahr)
Knochen und Weichteile sind atrophiert, deutliche Entkalkungen im
Röntgenbild sichtbar. Muskelatrophie, Funktionseinschränkung, die Haut
ist meist dünn und blass.

( Tab. 1). Sowohl die klinische Praxis als auch die Fachliteratur zeigen jedoch, dass es sich um ein Schmerzsyndrom mit fließenden Übergängen in Symptomatik und Ausprägung handelt. Eine Einteilung in Stadien kann demnach nur als pragmatischer Versuch angesehen werden [2]. In vielen Fällen kann diese Einteilung jedoch die Zuordnung zu einer entsprechenden handtherapeutischen Anwendung erleichtern.

In der modernen Therapie des CRPS hat sich ein multimodales Behandlungskonzept (MMST: multimodale Schmerztherapie) durchgesetzt. Neben der Physio- und Ergotherapie zählen die medikamentöse Therapie sowie die Psychotherapie zu den Basistherapieformen. Ergänzend zu diesen können je nach Verlauf Grenzstrangblockaden, Spinal Cord Stimulation oder intrathekale Injektionen mit Baclofen durchgeführt werden [3].

In den Leitlinien der Arbeitsgemeinschaft Wissenschaftlicher Medizinischer Fachgesellschaften (AWMF-Leitlinien, 2012) zum CRPS [3] wird ausdrücklich die intensive Anwendung von Physio- und Ergotherapie (hier im Weiteren als Handtherapie zusammengefasst) unter systemischer Analgesie und antineuropathischer Medikation als Basistherapie genannt jedoch nicht weiter spezifiziert.

So ist die Verordnung von Physiotherapie und Ergotherapie im klinischen Alltag häufig die erste therapeutische Maßnahme des Handchirurgen und viele Autoren betonen die Wichtigkeit der handtherapeutischen Maßnahmen im Rahmen der Behandlung des CRPS [2-6].

Es besteht jedoch das Problem, dass zum einen die Physio- als auch die Ergotherapie einen weit gefassten Begriff mit zahlreichen unterschiedlichen Anwendungen darstellen, zum anderen gleichartige Anwendungen unterschiedlich benannt oder auch ausgeführt werden können. Es fehlen also zum einen klare Definitionen der Begrifflichkeiten und zum anderen klare Spezifizierungen hinsichtlich der meist sehr allgemein gefassten Behandlungsempfehlungen.

Ein weiteres Problem ist, dass die einzelnen Therapiemaßnahmen innerhalb der Handtherapie bisher kaum wissenschaftlich untersucht und auf Ihre Wirksamkeit im Rahmen der Behandlung des CRPS nicht überprüft sind. So existiert im Zeitalter der „evidenz-basierten Medizin“ kein wissenschaftlich begründeter Behandlungsalgorithmus, bzw. erscheinen die wenigen, vorliegenden Therapierichtlinien [6] unvollständig oder veraltet.

Ziel dieser Arbeit ist es daher, die in der Behandlung des CRPS zur Anwendung kommenden handtherapeutischen Techniken hinsichtlich Begrifflichkeit, Funktion, Anwendung/Durchführung und Wirksamkeit darzustellen und falls möglich einen aktuellen Behandlungsalgorithmus daraus abzuleiten.

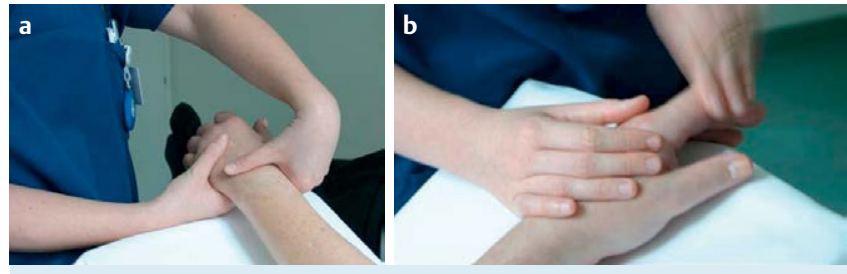

Abb. 1 Entstauende Schöpfgriffe der Lymphdrainage an Handrücken a und Finger $\mathbf{b}$.

\section{Handtherapeutische Behandlungsansätze beim CRPS}

Im Folgenden werden die unterschiedlichen, in der Behandlung des CRPS zur Anwendung kommenden, handtherapeutischen Maßnahmen vorgestellt und erläutert. Diese sollten im klinischen Alltag Bestandteil oben genannter MMST sein, welche häufig initial über einen Zeitraum von ca. 3 Wochen stationär verläuft [7]. Hieran folgt das ambulante Intervall mit Fortsetzung der intensiven multimodalen Behandlung.

\section{Lymphdrainage}

Die Lymphdrainage ist eine sanfte Massageformen mit dem Ziel der Ödemreduktion. Synonym werden auch die Begriffe Komplexe Physikalische Ödemtherapie (KPE), Manuelle Lymphdrainage (MLD) oder einfach Entstauungstherapie verwendet.

90\% der Lymphgefäße verlaufen dicht unter der Haut und sind somit manuell gut erreichbar [8]. Durch kreisende Druckimpulse wird eine Steigerung des Abflusses von interstitieller Flüssigkeit erreicht ( $\bullet$ Abb. 1). Die Motorik der Lymphgefäße wird gesteigert, indem die Pumpleistung der Lymphgefäße beeinflusst wird. Unter Ruhebedingungen beträgt die Frequenz der Lymphgefäße ca. 10-12 Kontraktionen pro Minute, dies kann mithilfe der Therapie auf bis zu 20 gesteigert werden [9]. Die Grifftechniken werden mit wechselndem Druck angewendet, dabei kommt es in der Druckphase zu einer Quer- bzw. Längsdehnung, was einen Reiz für das Gewebe darstellt. Die glatten Muskelzellen der Lymphgefäße reagieren auf diesen Reiz mit einer Steigerung der Pumpfrequenz. Einer oftmaligen Wiederholung der Griffe folgt eine erhöhte Durchflussrate. Neben der entstauenden Wirkung kommt es zu einer sympathikolytischen Wirkung mit Tonussenkung der Skelettmuskulatur [9].

\section{Manuelle Therapie}

Unter Manueller Therapie versteht man die Befundaufnahme und ggf. Therapie von Gelenkfunktionsstörungen. Diese Therapieform wird mit dem Ziel der Bewegungserweiterung und Gelenksentlastung durchgeführt.

In der Manuellen Medizin oder Manuellen Therapie wird ein Gelenk mit allen Einflussfaktoren als Funktionseinheit betrachtet. Alle artikulären und periartikulären anatomischen Strukturen werden hinsichtlich ihrer pathophysiologischen Auswirkung auf die Gelenkdysfunktion untersucht und wenn nötig therapiert [10]. Das Spektrum der manuellen Techniken umfasst verschiedene Mobilisations- und Dehnverfahren sowie muskulär wirksame Techniken, wie z.B. die Triggerpunkttherapie, die myofaszialen Release-Techniken, reflektorisch unterstützende Therapieformen und verschiedene Massagetechniken ( $\bullet$ Abb. 2). Passiv geführte Bewegungen und die Unterstützung aktiver Bewe- 

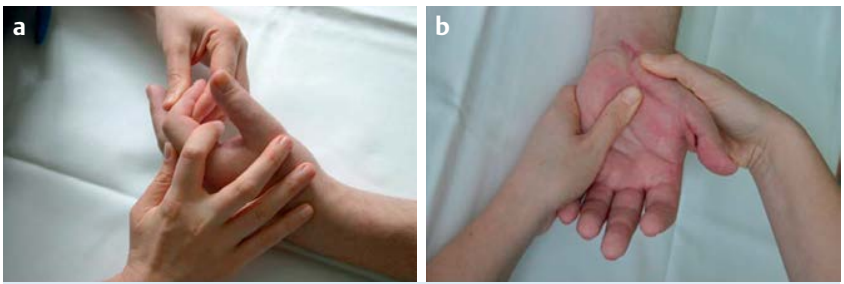

Abb. 2 Mobilisationstechniken am Zeigefingermittelgelenk a und der Hohlhand $\mathbf{b}$.

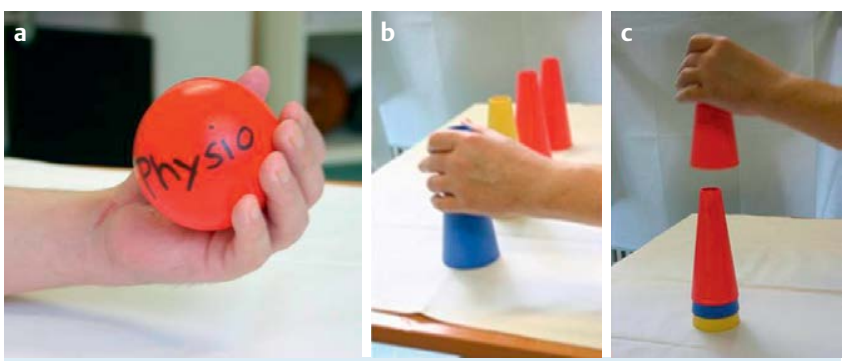

Abb. 3 Aktives Üben mit Geräten. Der Einsatz eines Gymnastikballs a. Koordinationsübung zur zielgerichteten aktiven Bewegung b, c.

gungsmuster (assistives Bewegen) runden das Bild der manuellen Therapieformen $a b$.

Schmerzen können im Allgemeinen durch Aktivierung inhibitorischer Interneurone der Hautpropriozeptoren sofort beeinflusst werden [10]. So soll durch die Manuelle Therapie eine Downregulation von Wide-Dynamic-Range Neuronen erfolgen, die über zahlreiche propriozeptiven A-Beta-Afferenzen mit inhibitorischen GABAergen Interneuronen verfügen und eine Hemmung des nozizeptiven Reflexbogens erzielen. Eine chronifizierte neurogene Entzündung führt zu einer Verkleinerung der inhibitorischen und einer Verstärkung der nozizeptiven Felder. Daher sollte vor dem Einsatz der Manuellen Therapie ein medikamentöses Regime zur Reduktion der neurogenen Entzündung begonnen werden. Inwieweit die inhibitorischen Felder beim CRPS noch vorhanden sind bzw. der Chronifizierungsprozess im zentralen Nervensystem fortgeschritten ist, entscheidet über die Wahl der segmental angewandten Manuellen Therapie [11].

\section{Bewegungstherapie/Aktive Bewegung/}

Krankengymnastik

Im Gegensatz zu der Manuellen Therapie, die meistens für die Patienten passiv durchgeführt wird, steht bei der Bewegungstherapie das aktive Üben der gestörten Funktion im Fokus ( $\bullet$ Abb. 3). Ziele des aktiven Bewegens sind Wiederherstellung der eingeschränkten Funktion, Muskelkräftigung, Koordinationsverbesserung, Beseitigung von Sensibilitätsstörungen und das übergeordnete Ziel, die Erhaltung der Selbstständigkeit der Patienten. Sämtliche Bewegungsrichtungen sollen beim aktiven Üben berücksichtigt werden. Eine besondere Betonung liegt zwar auf dem Bewegen in die eingeschränkten Bewegungsrichtungen, aber nicht ausschließlich. Jedes Gelenk soll endgradig bewegt werden. Die Intensität richtet sich nach dem Schmerz und der Funktionsfähigkeit. Schmerzen und reflektorische Abwehrspannungen sollten konsequent vermieden werden. Die aktiven Übungen sind auch als Kombination mit der Spiegeltherapie (s. unten) durchführbar.

Darüber hinaus sollten auch die angrenzenden Gelenke mit einbezogen werden. Die Intensität muss individuell angepasst wer- den, aber auch das Üben gegen Widerstand (resistives Üben) gehört zur aktiven Bewegung und dient der Kräftigung und dem Muskelaufbau.

\section{Propriozeptive neuromuskuläre Fazilitation (PNF)}

Die propriozeptive neuromuskuläre Fazilitation, im folgenden PNF genannt, kann als Sonderform der Bewegungstherapie angesehen werden. Ziel dieser Therapie ist die Fazilitation (Anbahnung) gestörter Bewegungsmuster.

Bei der PNF werden sowohl Exterozeptoren, Telerezeptoren (hier vor allem Augen und Ohren) und vor allem Propriozeptoren genutzt, um physiologische, funktionelle und koordinierte Bewegung anzubahnen. Ziel bei der PNF-Bewegungstherapie ist es, durch verstärkte Stimulation der Sensoren das neuromuskuläre Zusammenspiel zu fördern und damit physiologische Bewegungsmuster zu ermöglichen (Fazilitation), die im Zentralnervensystem abgespeichert sind [12].

Praktisch werden 3-dimensionale, physiologische Bewegungsmuster an einem Körperabschnitt gegen einen angepassten Widerstand durchgeführt, meist mit einer rotatorischen Komponente. Hintergrund ist, dass sich funktionelle Bewegungsmuster immer durch Dreidimensionalität auszeichnen. Bei den Bewegungsmustern der Extremitäten ist grundsätzlich auch eine flektierende oder extensorische Komponente des Ellbogens bzw. des Kniegelenks zu finden. Die Bewegungen werden mit festgelegten taktilen Reizen durchgeführt. Die Patienten initiieren die Bewegung aus einer korrekten Vordehnung des Muskels gegen einen angepassten 3-dimensionalen Widerstand bis in die Endstellung des Bewegungsmusters [12]. Das Zentralnervensystem speichert komplexe Bewegungsmuster wie z.B. das Gangmuster oder auch die Greiffunktionen der Hand. Die summierten Reize des Bewegungsmusters werden über das periphere Nervensystem zum zentralen Nervensystem (ZNS) geleitet. Sobald ein Teil des komplexen Bewegungsmusters erkannt wird, sendet das Zentralnervensystem die entsprechenden Informationen für die Muskelaktivität an alle anderen beteiligten Körperabschnitte. Diese Weiterleitung der Aktivität in andere Körperabschnitte wird physiologische Irradiation genannt. Dieser Mechanismus stellt die Grundlage für die Fazilitation einer physiologischen Bewegung dar [12].

\section{Graded Motor Imagery und Spiegeltherapie}

Das Graded Motor Imagery (GMI) und damit auch die Spiegeltherapie, die ein Bestandteil davon ist, kann als Teil des mentalen Trainings angesehen werden [13,14]. Ziel dieser Therapie ist Bewegungs- bzw. Funktionsförderung und Schmerzreduktion mithilfe der Vorstellungskraft und im Falle der Spiegeltherapie mit einer starken, optischen Stimulation ( $\bullet$ Abb. 4).

Die Grundlage der Therapie basiert auf der Annahme, dass mittels Aktivierung der prä- und primärmotorischen Kortexareale simultan mit den zugehörigen sensorischen Arealen eine kortikale Reorganisation erreicht wird. Man unterscheidet 3 Phasen der Anwendung [13-15].

1. Phase: Darstellung von verschiedenen Händen, bei denen die Lateralität erkannt werden muss. Messparameter sind hier Geschwindigkeit und Treffsicherheit. Es wird der prämotorische Kortex aktiviert [13].

2. Phase: Betrachtung von verschiedenen Handpositionen, die mental eingenommen werden. Führt zu einer Aktivierung des primären motorischen und sensorischen Kortex.

3. Phase: Spiegeltherapie. Die Patienten beobachten Bewegungen der nichtbetroffenen Hand 4 min lang im Spiegel und 


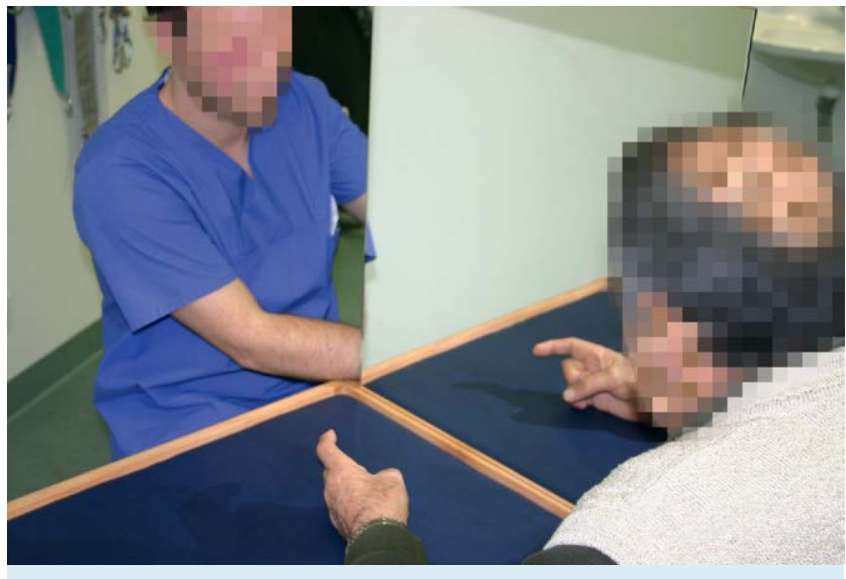

Abb. 4 Anwendung der Spiegeltherapie bei erkrankter rechter Hand.

stellen sich vor, die Bewegung auch mit der betroffenen Hand durchzuführen. In einem zweiten Schritt wird die Bewegung soweit möglich tatsächlich mit der betroffenen Hand hinter dem Spiegel ausgeführt. Es werden alle kortikalen motorischen und sensorischen Areale aktiviert [13-15].

\section{Physikalische Maßnahmen \\ Kohlensäure-Bad}

Beim CRPS und in der Rheumatologie werden normalerweise Teilbäder der betroffenen Extremität, gelegentlich auch der nicht-betroffenen Extremität verwendet. Letzteres ist in jedem Stadium möglich. Ziel der Anwendung von $\mathrm{CO}_{2}$-Bädern ist eine Reduktion der Schmerzsymptomatik. Daneben soll eine Ödemreduktion und eine Verbesserung der Beweglichkeit erzielt werden $[5,16]$. Diesen Effekt erklärt man sich unter anderem durch die Vasodilatation nach Kohlensäurebad, welche einen Anstieg der Mikrozirkulation und des transkutanen Sauerstoffpartialdrucks sowie eine Absenkung des arteriellen Blutdrucks zur Folge hat $[5,16]$.

Die Temperatur sollte beim CRPS phasendifferenziert gewählt werden. Klassischerweise werden in der Frühphase des CRPS kühle $\mathrm{CO}_{2}$-Bäder gewählt, mit einer Temperatur von $27-32^{\circ}$ für max. $20 \mathrm{~min}$. Dies hat einen analgetischen und antiödematösen Effekt [5]. In den folgenden Phasen hingegen sollte das $\mathrm{CO}_{2}$-Bad nach Mucha eine Temperatur von $39^{\circ}$ haben [5]. Bei beidseitiger Anwendung kommt die konsensuelle Reaktion als zusätzlicher positiver Effekt hinzu.

\section{Thermische Therapie}

Thermische Therapien werden zu verschiedenen Zwecken angewandt. Ammer [17] definiert die Thermotherapie als „Die Zufuhr oder der Entzug von Wärmeenergie, um Behandlungseffekte zu erzielen“.

Sowohl bei Kälte- als auch bei Wärmetherapien kommt es zu einer Vasodilatation und damit zu einer erwünschten Mehrdurchblutung. Bei der Kältetherapie tritt allerdings zunächst eine Vasokonstriktion auf, die reflektorisch dann dilatierend nachwirkt, die sog. reaktive Hyperämie [6]. Der Wärmetherapie wird eine schmerzlindernde und tonussenkende Wirkung attestiert, insbesondere wenn es sich um muskuläre Beschwerden handelt. Der Kältetherapie wird eine schmerzlindernde und entzündungshemmende Wirkung zugeschrieben [6].
TENS

Auf der Grundlage der Gate-Control-Theory von Melzack und Wall [18] wurde Anfang der 70er Jahre eine Methode entwickelt, die niedrige Spannungsmodulationen über epikutane Silikonelektroden appliziert, die Transcutaneous Electrical Nerve Stimulation, kurz TENS. Ziel des TENS ist die Schmerzlinderung. Dies geschieht durch Minimierung oder sogar komplette Ausschaltung von dicken myelinisierten afferenten Nervenfasern (A-Beta-Fasern), die Transmission nozizeptiver Inputs über Aktivierung der schwach myelinisierte (A-Delta-Fasern) und nicht-myelinisierte sympathische Afferenzen (C-Fasern) unterdrücken [19].

\section{Ergotherapie}

Die Ergotherapie ist definiert als eine Therapieform, die sich mit der Ausführung konkreter Betätigungen und deren Auswirkungen auf den Menschen und dessen Umwelt befasst. Beeinträchtigungen werden durch den gezielten Einsatz von individuell sinnvollen Tätigkeiten behandelt. Die Ziele der Ergotherapie sind vielfältig und reichen über den Erhalt der Funktion und der Selbstständigkeit bis hin zur Schmerzlinderung. Entsprechend dieser weit gefassten Definition und der großen Anzahl unterschiedlicher Methoden sind auch die ergotherapeutischen Anwendungen bei der CRPS Behandlung nicht exakt beschrieben. Im engeren Sinne kann man evtl. hier die Schulung der Alltagsaktivitäten, den Bau von speziellen Schienen und die sensomotorische Stimulation unter Ergotherapie zusammenfassen.

Zunächst erfolgt, allerdings nur wenn notwendig, die Erstellung von Lagerungsschienen und ggf. auch Maßnahmen zum Gelenkschutz, vor allem wenn die Patienten Begleiterkrankungen aus dem rheumatischen Formenkreis haben.

Darüber hinaus erfolgt ab dem Stadium I die Behandlung der Hyperalgesie durch sensorische Stimulation. Beim CRPS finden Veränderungen auf kortikaler Ebene im Zentralnervensystem statt. Durch die Hyperalgesie und zum Teil Allodynie werden andere Fähigkeiten vernachlässigt, die für die Sensibilität und die Motorik der betroffenen Extremität existentiell sind [20]. Ziel der Ergotherapie ist es, diesen sog. Neglect des schmerzhaften Körperabschnittes im Gehirn (Gyrus prä- und postcentralis) zu mindern. Zu diesem Zweck wird die betroffene Extremität unterschiedlichen sensiblen Reizen ausgesetzt, wie bspw. mit Wasser oder Watte berührt oder in eine Schüssel mit Linsen, Reis, Zucker, Erbsen und ähnliches gelegt und wenn möglich darin bewegt. Damit soll schrittweise ein normales Empfindungsvermögen wiedererlangt werden. Mit zunehmendem Fortschritt und abnehmenden Schmerz bewältigen die Patienten sensomotorisch anspruchsvollere Aufgaben, wie das Identifizieren einfacher Gegenstände durch den Tastsinn (Stereognosie).

Ein weiteres Therapieziel ist die Erhaltung der persönlichen Selbstständigkeit und wenn möglich die Wiedereingliederung in den Beruf. Dazu wird ein Selbsthilfetraining durchgeführt (Schulung der Aktivitäten des Alltags/Activity of daily life=ADL), und oftmals muss, insbesondere bei älteren Patienten, eine Versorgung mit einfachen Hilfsmitteln erfolgen (Anziehhilfen, Schreibhilfen, rutschfeste Unterlagen, Küchenutensilien, wie z.B. angepasste Dosenöffner, Flaschenöffner usw.). Bei nachlassender Schmerzintensität kommen redressierende Maßnahmen wie eine Quengelschienung und dynamische Bewegungsschienen, Intensivierung von Fein- und Grobmotorikschulung sowie leichte Tätigkeiten gegen zunehmenden Wiederstand zum Einsatz [20]. 
Wichtig ist bei allen therapeutischen Maßnahmen, dass sie keinesfalls zu einer Schmerzintensivierung führen dürfen.

\section{Diskussion}

Das CRPS im klinischen Vollbild gilt im handchirurgischen Alltag zwar als selten, wenn es jedoch auftritt, ist es von hoher sozioökonomischer Relevanz. Darüber hinaus mag das klinische Vollbild zwar eher selten zu beobachten sein, dennoch treten in der Praxis relativ häufig dystrophe Verlaufsformen nach Trauma oder Operation auf, die eine ähnliche Behandlungsstrategie auf handtherapeutischem Gebiet verlangen oder zumindest nahelegen.

So steht die Handtherapie im klinischen Alltag häufig an erster Stelle eines komplexen Behandlungskonzepts und ist für die Funktionalität der Hand nach abgeschlossener operativer Behandlung von entscheidender Bedeutung. Dieser Stellenwert spiegelt sich jedoch bei Betrachtung der Literatur nicht wieder. Im Themenheft „CRPS“ der Zeitschrift für Handchirurgie, Mikrochirurgie und Plastische Chirurgie vom Februar 2010 bspw. finden sich Beiträge über die Stadieneinteilung und die Klinik, über die Pathophysiologie, über Differenzialdiagnosen und über die medikamentöse Therapie des CRPS. Ein Artikel über die physiound ergotherapeutischen Behandlungsmöglichkeiten beim CRPS fehlt. Eventuell spiegelt dies eine fehlende Vernetzung zwischen Ärzten und Therapeuten oder allgemein ein fehlendes Interesse der Ärzte an der Handtherapie wieder? Diese könnte auch die geringe Anzahl an aussagekräftigen wissenschaftlichen Untersuchungen hinsichtlich handtherapeutischer Anwendungen beim CRPS erklären. So ist die Studienlage betreffend handtherapeutischer Interventionen im Vergleich zu anderen medizinischen Bereichen äußerst dürftig, selbst wenn das Evidenzlevel dabei nicht berücksichtigt wird. Die AWMF-Leitlinie zur Behandlung des CRPS bspw. beschreibt die Ergo- und Physiotherapie als Basistherapie, bezieht sich dabei jedoch lediglich auf 2 Studien derselben Forschergruppe, welche Physiotherapie und Ergotherapie gegeneinander verglichen haben [21,22]. Ergebnis war, dass die Physiotherapie geringfügig bessere Ergebnisse bezüglich der Reduktion von Schmerz und Beweglichkeit erzielte als die Ergotherapie.

Die Manuelle Therapie wird von der deutschen Gesellschaft für Physikalische Medizin und Rehabilitation ab Stadium 2 des CRPS bei zunehmender Bewegungseinschränkung empfohlen. Im Stadium 3 ist die Manuelle Therapie zur Mobilisierung der Gelenke uneingeschränkt durchzuführen. Letzten Endes beruht diese Empfehlung jedoch lediglich auf Einzelfallberichten bzw. Expertenerfahrung [23-25]. Diese beschreiben alle eine Verbesserung der Schmerzintensität und der Beweglichkeit bei Ihren Patienten. Ein Review der Untersucher Muir und Vernon [26] von 2000 beschreibt ebenfalls die mangelnde Anzahl an kontrolliert randomisierten Studien, welche die Wirksamkeit der manuellen Therapie beim CRPS untersuchen.

Die aktive Beübung hingegen ist vergleichsweise gut untersucht, da in den meisten Studien die aktive Bewegung in den physiotherapeutischen Behandlungsprotokollen stets integriert ist. Insgesamt 5 Studien [21,22,27-29] - alle mit prospektiv randomisiertem Design - konnten die Wirksamkeit der aktiven Bewegung bei CRPS hinsichtlich Schmerzreduktion und Funktionsverbesserung belegen.

Oerlemans und Kollegen konnten in 2 Studien [21,22] die Vorteile der aktiven Physiotherapie bei CRPS-Patienten aufzeigen.
Auch von Wu et al. wurde bereits 1999 ein positiver Effekt von Ganzkörperbewegungen wie dem QiGong nachgewiesen [29]. Allerdings postulierten sie auch einen negativen Effekt von übermäßigem Training, eine Aussage, welche sich nicht mit den Ergebnissen von Sherry et al. deckt [28]. In ihrer prospektiven Studie untersuchten diese 103 Jugendliche mit CRPS. Diese mussten 4 Stunden täglich an einem intensiven Bewegungsprogramm für den ganzen Körper teilnehmen. Es wurde keine pharmakologische Intervention durchgeführt. Bei allen wurde eine psychologische Beurteilung durchgeführt, 77\% der Jugendlichen wurden aufgrund dieser Beurteilung psychotherapeutisch begleitet. $92 \%$ der Jugendlichen erreichten eine deutliche Schmerzlinderung und volle Funktionsfähigkeit. Das Follow-up nach 2 Jahren konnte nur an 49 Probanden durchgeführt werden, von diesen waren $88 \%$ nach wie vor beschwerdefrei.

Studien speziell zur Sicherung der Wirkungsweise und Wirksamkeit der PNF bei CRPS-Patienten existieren nicht. Dennoch kann PNF schmerzadaptiert zur Aktivierung der betroffenen Extremität angewendet werden und damit eine drohende Immobilisierung vermieden werden.

Die Wirksamkeit der Spiegeltherapie ist mittlerweile vergleichsweise gut belegt. Zahlreiche Studien bestätigen die Wirksamkeit der Spiegeltherapie bei CRPS-Patienten im akuten Stadium hinsichtlich Reduktion der Schmerzintensität und Verbesserung von Funktion und Perzeption $[2,9,14,15,30]$. So hat die Spiegeltherapie mittlerweile einen festen Stellenwert, sowohl in der Therapie des CRPS als auch generell in der Behandlung von neuropathischen Schmerzen oder Phantomschmerzen [30]. So sollte nach der aktuellen Literatur die Anwendung so früh wie möglich in Stadium 1 begonnen und mehrfach täglich in kurzen Behandlungseinheiten wiederholt werden. Eine mögliche Langzeitwirkung der Spiegeltherapie konnte bisher allerdings nicht nachgewiesen werden. Hier bedarf es aus unserer Sicht weiterer Studien.

Die Wirksamkeit der manuellen Lymphdrainage bei CRPS wurde bisher in 3 Studien untersucht. 2 Studien hatten ein randomisiert kontrolliertes Design [31-33]. Nur in einer dieser Studien konnte für die Akutphase eine signifikante Ödemreduzierung durch die 2-monatige Anwendung von Lymphdrainage dargestellt werden. Allerdings konnte bereits bei der Messung weitere 2 Monate nach der Behandlung kein statistisch signifikanter Unterschied mehr festgestellt werden. Bei der Einzelfallstudie wurden „positive Reaktionen“ auf die Behandlung durch Lymphdrainage beschrieben, allerdings ohne Berücksichtigung des Langzeiteffektes.

Additive Maßnahmen innerhalb der Handtherapie wie die physikalischen Anwendungen sind vergleichsweise schlecht untersucht bzw. deren Nutzen bei der Behandlung des CRPS nur selten wissenschaftlich erwiesen. Dies liegt jedoch unter anderem auch daran, dass häufig die Wirkung einzelner Maßnahmen generell bekannt und mit Studien belegt, nur die Anwendung speziell beim CRPS nicht untersucht ist.

Ausnahme hierbei ist das Kohlensäurebad. Mucha und Kollegen konnte 2005 durch eine Studie mit 34 Patienten nachweisen, dass ein $\mathrm{CO}_{2}$-Bad bei der Behandlung des CRPS zusätzlich zur obligatorischen Physiotherapie signifikant positive Effekte auf die Parameter Schmerzentwicklung, Ödemreduktion und Beweglichkeit hat $[5,16]$.

Der Einsatz thermischer Therapie bei CRPS hingegen wurde bisher im Wesentlichen theoretisch diskutiert und daher lediglich als Empfehlung ausgesprochen. So sollte die Wärmeapplikation 
in der akuten Phase des CRPS vermieden werden, da Wärme bei manchen entzündlichen Prozessen die Ödembildung verstärken kann und die Patienten im Allgemeinen eine deutliche Wärmeunverträglichkeit aufweisen [6].

Hinsichtlich der Kältetherapie gibt es lediglich eine Untersuchung von Ammer und Kollegen [17], die eine Hochvoltbehandlung mit oder ohne Kryotherapie kombiniert haben. Eingeschlossen wurden 29 Patienten mit algodystrophen Ödemen. Diese Untersuchung ergab keinen signifikanten Unterschied. So finden sich aktuell keine weiteren Studien, die den Einsatz von Kältetherapie dringend nahelegen. Im Gegenteil, aus heutigem Verständnis der Schmerztherapie wird vom Einsatz starker Kältereize grundsätzlich abgeraten. Dies bestätigt eine Arbeit von 2002 zur Ganzkörperkühlung aus der Rheumatologie, die zeigt, dass über den Sympathikusreflex Schmerzen in der Hand ausgelöst werden können [34]. So sollte ein starkes Absenken der Gewebstemperatur mittels Eis und Eispackungen auf jeden Fall vermieden werden, da es zu einer reflektorischen Vasodilatation mit Schmerz- und Ödemverstärkung kommen kann [6].

Was die Wirksamkeit von TENS beim CRPS angeht, gibt es nur wenige Daten, die den positiven Effekt belegen. Robaina et al. [35] verglichen 1989 in ihrer Studie den Einsatz von TENS allein mit dem Einsatz von TENS und Spinal Cord Stimulation. In dieser Arbeit wurde bei 18 der 35 eingeschlossen Patienten eine sehr gute oder gute Schmerzreduktion durch TENS allein erzielt. Sie kamen darüber hinaus zu dem Ergebnis, dass beide Therapien bessere Resultate erzielten als Sympathikusblockaden und Grenzstrangresektionen. Auch bei anderen Krankheitsbildern wird die Wirksamkeit des Einsatzes von TENS kontrovers diskutiert [36]. Die insgesamt dürftige Studienlage erlaubt keine abschließende Beurteilung der Wirksamkeit.

Obwohl die Ergotherapie eine der Basismaßnahmen innerhalb der Handtherapie beim CRPS darstellt, gibt es keine größeren klinischen Studien hierzu. Die schmerzreduzierende Wirkung der Ergotherapie wurde bisher lediglich durch eine Studie des Forschungsnetzwerkes „Neuropathischer Schmerz“ untersucht [37]. Eingeschlossen wurden 6 Patienten, die 6 Monate ein Trainingsprogramm wie unter Punkt 2,6 beschrieben absolvierten und bei denen eine Messung der neuronalen Aktivität durchgeführt wurde. Eine Kontrollgruppe gab es nicht. Ergebnis nach 6-monatigem Training war eine deutliche Zunahme der anfänglich stark reduzierten Aktivität der Nervenzellen, und es konnte eine drastisch verringerte durchschnittliche Schmerzstärke festgestellt werden [20].

Generell fordern wir im Zeitalter der evidenz-basierten Medizin einen - möglichst mittels prospektiv angelegten, randomisierten kontrollierten Studien - wissenschaftlich belegten Vorteil unserer Therapie. Dies ist grundlegend auch richtig. Es gibt jedoch Situationen - wie oben bereits angesprochen - in denen nicht auf die Aussage von mehreren solcher randomisierten, prospektiv angelegten Fallkontrollstudien zurück gegriffen werden kann. Dies sollte jedoch nicht dazu führen entweder keine Behandlung einzuleiten und davon auszugehen, dass die Behandlung keinen Erfolg haben kann oder die Behandlung unstandardisiert und willkürlich durchführen zu lassen.

Nach Sacketts [38] Definition der evidenz-basierten Medizin kann nämlich auch dann von evidenzbasierter Therapie gesprochen werden, wenn klassische randomisierte Kontrollstudien fehlen. Hiernach gilt evidenz-basierte Medizin als die Synthese der besten verfügbaren Nachweise aus der medizinischen Wissenschaft, der ärztlichen (therapeutischen) Berufserfahrung und den Wertevorstellungen des Patienten [38].
Tab. 2 Handtherapeutischer Behandlungsalgorithmus beim CRPS.

\section{Therapeutische Maßnahmen im Stadium I:}

- GMI und hochfrequente Spiegeltherapie (zwischen 7-14 mal täglich)

- Aktive Bewegungstherapie in schmerzangepasster Dosierung bzw. Intensität, PNF nur mit Führungswiderstand (gering)

- Ergotherapie:

- Sensorische Reize wie lauwarmes Wasser, feiner Sand, Raps o.ä.

- Hilfsmittels für den Alltag/Arbeitsplatz in jedem Stadium möglich und nötig

- generell redressierende Maßnahmen wie Schienen/Quengelbehandlung werden erst ab Stadium III empfohlen und nur bei individueller Notwendigkeit

- Kohlensäurebäder

- Lymphdrainage

Therapeutische Maßnahmen im Stadium II:

- Aktive Bewegungstherapie in schmerzangepasster Dosierung bzw. Intensität, jedoch nach Möglichkeit gesteigert, PNF mit leichtem, schmerzangepassten Widerstand

- GMI und hochfrequente Spiegeltherapie (zwischen 7-14 mal täglich), mit und ohne Materialien

- Manuelle Therapie unterhalb der Schmerzgrenze

- Kohlensäurenbäder

- Ergotherapie:

- Linsenbäder, Abhärtungen mit gröberen Materialien ab Stadium 2, abhängig von der Schmerzsituation

- Hilfsmittelversorgung, angepasst an die Fähigkeiten und Funktionen

Therapeutische Maßnahmen im Stadium III:

- Aktive Spiegeltherapie (zwischen 7-14 mal täglich) in Kombination mit vielen unterschiedlichen Materialien und sensorischen Reizen

- Aktive Bewegungstherapie in ansteigender Intensität, PNF mit ansteigendem Widerstand, Krafttraining

- Manuelle Therapie unterhalb der Schmerzgrenze

- Dehnungen zur Bewegungserweiterung

- Kohlensäurebäder

- Ergotherapie:

- Hilfsmittels für den Alltag wenn möglich reduzieren und an Alltag und Haushalt anpassen

- generell redressierende Maßnahmen wie Schienen/Quengelbehandlung nur bei individueller Notwendigkeit

- intensive Schulung der Fein- und Grobmotorik/Haltungschulung

So erscheint es uns möglich im Rahmen dieser Arbeit, basierend auf oben genannter Synthese aller 3 Bereiche (medizinische Wissenschaft, ärztliche/therapeutische Berufserfahrung und Wertevorstellung des Patienten), einen handtherapeutischen Behandlungsvorschlag für das CRPS zu entwickeln, welcher das Stadium und den individuellen Verlauf berücksichtigt ( $\bullet$ Tab. 2). Additive Maßnahmen wie TENS, Elektrotherapien, PNF und Steigerungen wie z.B. das Krafttraining müssen jeweils individuell patienten- und therapeutenabhängig abgewogen werden.

Leider erbringt die Studienlage meist keine Aussagen über Häufigkeit, Dauer und Intensität der einzelnen handtherapeutischen Anwendungen. Im klinischen Alltag bilden vielmehr organisatorische und ökonomische Faktoren die dafür entscheidenden Einflussgrößen. So ist in vielen Kliniken organisatorisch nicht mehr als 30 min Physiotherapie und 30 min Ergotherapie pro Tag möglich. Hier postulieren wir jedoch, dass die Patienten von einer Steigerung dieser Anwendungen auf mindestens 2-mal täglich Ergound Physiotherapie durchaus profitieren würden. Dies legen die Studienergebnisse von Sherry et al. [28] und neuere Forschungsergebnisse auf dem Gebiet der Pain Exposure Therapy (PEPT) [39] nahe. Hier konnte gezeigt werden, dass durch eine zunehmende Belastung mit gezielter Schmerzkontrolle ohne Analgetika eine 
Änderung der zentralen Deregulierung und kortikalen Repräsentation der betroffenen Extremität möglich ist [39].

Vergleicht man den in $\odot$ Tab. 2 dargestellten Behandlungsalgorithmus mit Therapieleitlinien der Deutschen Gesellschaft für Physikalische Medizin und Rehabilitation aus dem Jahre 2002 [6] lassen sich im Laufe der letzten Jahre folgende Unterschiede/ Entwicklungen festhalten:

Grundlegend wird die Anwendung von großflächiger Kälte heutzutage eher kritisch gesehen, da unter Umständen hierdurch ein CRPS überhaupt erst ausgelöst oder zumindest die bestehende Symptomatik verstärkt werden kann [34]. Als wichtige Säule in der Handtherapie hat sich das GMI und die hochfrequente Spiegeltherapie in allen 3 klinischen Stadien des CRPS etabliert. Die Elektrotherapie und die TENS werden zurückhaltender eingesetzt und als additive Maßnahmen verstanden.

Leider gibt es nur wenig neue Therapieansätze oder Forschungsvorhaben, was die Behandlung des CRPS angeht. Einen neueren Therapieansatz stellt hierbei die bereits angesprochene Pain Exposure Therapy (PEPT) dar [39]. Diese ist ein Übungsprogramm mit stufenweise zunehmender Belastung und Kontrolle des Schmerzverhaltens, jedoch unter gänzlichem Verzicht von Analgetikaeinsatz. Die autonome Deregulierung und die Änderungen der kortikalen Repräsentation der betroffenen Extremität sollen rückgängig gemacht werden. Damit soll, die aufgrund von Schmerzvermeidungsverhalten entstehende Immobilisierung aufgehoben werden. Die Patienten müssen bei dieser Methode lernen, den Schmerz zu verstehen und aktiv zu ignorieren. Darüber hinaus wird von den Patienten erwartet, selbst zu üben und das Erlernte aktiv in den individuellen Alltag zu integrieren. Aktuell läuft hierzu eine kontrollierte, randomisierte Studie, bei der die Wirkung von PEPT mit anderen Behandlungsstrategien verglichen wird $[13,39]$.

\section{Schlussfolgerung \\ $\nabla$}

Zusammenfassend zeigt sich, dass - auch bei eingeschränkter Studienlage - eine handtherapeutische Behandlung des CRPS standardisiert möglich ist. So können die unterschiedlichen handtherapeutischen Anwendungen spezifisch der entsprechenden Klinik zugeordnet werden. Es fehlen jedoch weiterhin klare Behandlungsvorgaben hinsichtlich Anwendungsdauer, -häufigkeit und -intensität. Zudem hängt die Behandlung und damit letzten Endes auch der Therapieerfolg stark von der Erfahrung des jeweiligen Handtherapeuten ab. So bestehen zwischen unterschiedlichen Handtherapeuten bei ein und demselben Patienten zum Teil große Unterschiede hinsichtlich der Auswahl einzelner Anwendungen, als auch in der Durchführung derselben. Daher sollten aus unserer Sicht Patienten mit CRPS nur an speziellen handchirurgischen Schwerpunktzentren mit entsprechend erfahrenen und speziell ausgebildeten Therapeuten und Handchirurgen behandelt werden.

Zudem wäre es für die Zukunft wünschenswert, dass neue Studien auf dem Gebiet gemeinsam von Handtherapeuten und Handchirurgen initiiert und weitere Forschungsvorhaben etabliert werden.

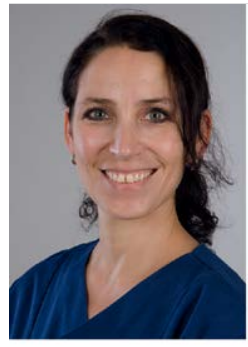

Corinna Körbler

geb. 1972 in Braunschweig, Besuch der Fachschule für Physiotherapie am Annastift Hannover 1993-1996. Mehrere Jahre Berufserfahrung in Hannover, anschließend Studium der Medizinalfachberufe an der Hochschule Hildesheim 2003-2005, mit dem Abschluss Bachelor of Science (BSc). Leitung der Physio- und Ergotherapeutischen Abteilung des Marienhospital Stuttgart seit 2005, nebenberufliches Masterstudium der Medizinalfachberufe an der Hochschule Hildesheim 2007-2010. Forschungsstipendium der Robert-Bosch-Stiftung im Internationalen Hospitationsprogramm, in diesem Rahmen 3 Monate Auslandsaufenthalt in Australien (Sydney Concord Hospital und Royal Perth Hospital) in der Plastischen und Verbrennungschirurgie. Seit September 2013 nebenberuflich Promovendin an der Eberhard Karls Universität in Tübingen.

\section{Interessenkonflikt: Nein}

\section{Literatur}

1 Harden RN, Bruehl S, Stanton-Hicks $M$ et al. Proposed new diagnostic criteria for complex regional pain syndrome. Pain Med 2007; 8: 326-331

2 Maihöfner C, Speck V. Graded Motor imagery for complex regional pain syndrome: Where are we now? European Journal Pain 2012; 16: 461-462

3 AWMF Leitlinienregister online. Leitlinien der Deutschen Gesellschaft für Neurologie. Leitlinien für Diagnostik und Therapie in der Neurologie. http://www.awmf.org/uploads/tx_szleitlinien/030-1161_S1_ Schmerzsyndrome_CRPS_2012-09.pdf

4 Carden E. Reflex Sympathetic Dystrophy: Recognition and Management for the Physician 2005; 1-2 http://www.forgrace.org/docu ments/carden-nonphysician.pdf

5 Stengg B, Prager C, Ammer K. Komplexes Regionales Schmerzsyndrom (CRPS 1 und 2). ÖZPMR, Österr. Z Phys Med Rehabil 2003; 13: 53-62

6 Werner GT, Beyer A, Berliner M et al. Therapierichtlinie der Deutschen Gesellschaft für Physikalische Medizin und Rehabilitation - Behandlung beim komplexen regionalen Schmerzsyndrom (sympathische Reflexdystrophie). Phys Med Rehab Kuror 2002; 12: 208-210

7 Kahl-Collins C. Physical Therapy Management of CRPS I Counterstrain: A Case Report. Journal of Manipulative Therapy 2007; 15: 25-41

8 Kraft $K$, Stange $R$. Lehrbuch Naturheilverfahren. Hippokrates Verlag, Stuttgart: 2010; 238-240

9 Zsusmann F, Moog-Egan M. Physiotherapie bei Schmerzproblemen. In: Frans van den Berg, Hrsg. Angewandte Physiologie Band 4. Thieme, Stuttgart: 2003

10 Heinmann D, Lawall J. Leitfaden Manuelle Therapie. 2005

11 Böhni U. Manuelle Medizin und Schmerz. Manuelle Medizin 2006; 15: $1-3$

12 Burton L, Brigham $H$. Propriozeptive neuromuskuläre Fazilitation. Eine Grundlage des funktionellen Trainings. http://www.functionalmo vementsystems.de/ARTIKEL/PropriozeptiveNeuromuskul\%C3\%A4re Fazilitation.aspx

13 Wendling-Hosch U. Den Schmerz ignorieren. Neue Erkenntnisse bei CRPS. Ergopraxis 2013; 6: 18-21

14 Neuro orthopadic institute. http://www.gradedmotorimagery.com/

15 Daly AE, Bialocerkowski AE. Does evidence support physiotherapy management of adult Complex Regional Pain Syndrome Type One? A systematic review. European Journal of Pain 2009; 13: 339-335

16 Mucha C. Einfluss von CO2-Bädern im frühfunktionellen Therapiekonzept der Algodystropie. Phys Rehab Kur Med 1992; 2: 173-178

17 Ammer $K$. Therapie posttraumatischer Veränderungen. In: Rathkolb $\mathrm{O}$, Ammer K, (Hrsg). Elektrotherapie (Hochvoltstimulation) bei Schmerzsyndromen. VLG, Berlin: 1989; 89-97

18 Melzack R, Wall PD. Pain mechanisms: a new theory. Science 1965 150: 971-979 
19 Emrich 0. (2001): Zeitschrift für angewandte Schmerztherapie http:// www.schmerz-therapie-deutschland.de/pages/zeitschrift/z4_01/ art_414.html

20 Maier C, Tegenthoff M. CRPS: Wenn schon ein Wattebausch Schmerz verursacht. Bochumer Mediziner entwickeln Training gegen Nervenschmerz: Annals of Neurology: Patienten lernen wieder fühlen. http:// www.pm.ruhr-uni-bochum.de/pm2005/msg00085.htm

21 Oehrlemans HM, Oostendorp RA, de Boo T et al. Pain and reduced mobility in complex regional pain syndrome I: outcome of a prospective randomised controlled clinical trial of adjuvant physical therapy versus occupational therapy. Pain 1999; 83: 77-83

22 Oerlemans HM, Goris JA, de Boo T et al. Do physical therapy and occupational therapy reduce the impairment percentage in reflex sympathetic dystrophy? Am J Phys Med Rehabil 1999; 78: 533-539

23 Celik D, Demirhan M. Physical Therapy and Rehabilitation of Complex Regional Pain Syndrome in Shoulder Prothesis. Korean J Pain 2010; 23: $258-269$

24 Shearer HM, Trim A. An unusual presentation and outcome of complex regional pain syndrome: a case report. J Can Chiropr Assoc 2006; 50: $20-26$

25 Bortolotto J. Reflex sympathetic dystrophy: an enigmatic improvement with spinal manipulation. J Can Chiropr Assoc 2000; 44: 245-251

26 Muir JM, Vernon H. Complex regional pain syndrome and chiropractic. J Manipulative Physiol Ther 2000; 23: 490-497

27 Oehrlemans HM, Oostendorp RA, de Boo T et al. Adjuvant physical therapy versus occupational therapy in patients with reflex sympathetic dystrophy/complex regional pain syndrom type I. Arch Phys Med Rehabil 2000; 81: 49-56

28 Sherry $D$, Wallace CA, Kelley $C$ et al. Short- and Long-term Outcomes of Children with Complex Regional Pain Syndrome Type I Treated with Exercise Therapy. The Clinical Journal of Pain 1999; 15: 218-223

$29 \mathrm{Wu} W H$, Bandilla E, Ciccone DS et al. Effects of qigong on late-stage complex regional pain syndrome. Altern Ther Health Med 1999; 5: $45-54$
30 Ramachandran VS, Altschuler EL. The use of visual feedback, in particular mirror visual feedback, in restoring brain function. Brain 2009; 132: $1693-1710$

31 Duman I, Ozdemir A, Tan AK et al. The efficacy of manual lymphatic drainage therapy in the management of limb edema secondary to reflex sympathetic dystrophy. Rheumatol Int 2009; 29: 759-763

32 Uher EM, Vacariu G, Schneider B et al. Comparison of manual lymphdrainage with physical therapy in complex regional pain syndrome, type I. A comparative randomized controlled therapy study. Wien Klin Wochenschr 2000; 112: 133-137

33 Safaz I, Tok F, Taskayanatan MA et al. Manual lymphatic drainage in management of edema in a case with CRPS: why the(y) wait? Rheumatol Int 2011; 31: 387-390

34 Baron $R$, Binder A, Ulrich $W$ et al. Komplexe regionale Schmerzsyndrome. In: Schmerz 2003; 17: 213-226

35 Robaina FJ, Rodrigues JL, de Vera JA et al. Transcutaneous electrical nerve stimulation and spinal cord stimulation for pain relief in reflex sympathetic dystrophy. Stereotact Funct Neurosurg 1989; 52: 53-62

36 Nnoaham KE, Kumbang J. Transcutaneous electrical nerve stimulation (TENS) for chronic pain. In: Cochrane Database Syst Rev 2008; 3: 231-258

37 Pleger $B$, Tegenthoff $M$, Ragert $P$. Sensorimotor retuning in complex regional pain syndrome parallels pain reduction. Ann Neurol 2005; 57: 425-429

38 Sackett $D$. Evidence based medicine: what it is and what it isn't. British medical journal 1996; 312: 71-72

39 EkJW, van Gijn JC, Samwel H et al. Pain exposure physical therapy may be a safe and effective treatment for longstanding complex regional pain syndrome type 1: a case series. Clin Rehabil 2009; 23: 1059-1066 